\title{
Reverse vaccinology assisted designing of multiepitope-based subunit vaccine against SARS-CoV-2
}

\author{
Muhammad Tahir ul Qamar ${ }^{1 \dagger}$, Farah Shahid ${ }^{2 \dagger}$, Sadia Aslam³ ${ }^{3}$ Usman Ali Ashfaq ${ }^{2 *}$, Sidra Aslam², Israr Fatima ${ }^{2}$,
} Muhammad Mazhar Fareed ${ }^{2}$, Ali Zohaib ${ }^{4}$ and Ling-Ling Chen ${ }^{1 *}$

\begin{abstract}
Background: Coronavirus disease 2019 (COVID-19) linked with severe acute respiratory syndrome coronavirus 2 (SARS-CoV-2) cause severe illness and life-threatening pneumonia in humans. The current COVID-19 pandemic demands an effective vaccine to acquire protection against the infection. Therefore, the present study was aimed to design a multiepitope-based subunit vaccine (MESV) against COVID-19.

Methods: Structural proteins (Surface glycoprotein, Envelope protein, and Membrane glycoprotein) of SARS-CoV-2 are responsible for its prime functions. Sequences of proteins were downloaded from GenBank and several immunoinformatics coupled with computational approaches were employed to forecast B- and T- cell epitopes from the SARS-CoV-2 highly antigenic structural proteins to design an effective MESV.

Results: Predicted epitopes suggested high antigenicity, conserveness, substantial interactions with the human leukocyte antigen (HLA) binding alleles, and collective global population coverage of 88.40\%. Taken together, 276 amino acids long MESV was designed by connecting 3 cytotoxic T lymphocytes (CTL), 6 helper T lymphocyte (HTL) and 4 B-cell epitopes with suitable adjuvant and linkers. The MESV construct was non-allergenic, stable, and highly antigenic. Molecular docking showed a stable and high binding affinity of MESV with human pathogenic toll-like receptors-3 (TLR3). Furthermore, in silico immune simulation revealed significant immunogenic response of MESV. Finally, MEV codons were optimized for its in silico cloning into the Escherichia coli K-12 system, to ensure its increased expression.
\end{abstract}

Conclusion: The MESV developed in this study is capable of generating immune response against COVID-19. Therefore, if designed MESV further investigated experimentally, it would be an effective vaccine candidate against SARS-CoV-2 to control and prevent COVID-19.

Keywords: SARS-CoV-2, COVID-19, Structural protein, Epitope, Vaccine, Multiepitope-based subunit vaccine, Immunoinformatics

\footnotetext{
*Correspondence: usmancemb@gmail.com; Ilchen@mail.hzau.edu.cn

${ }^{+}$Muhammad Tahir ul Qamar and Farah Shahid contributed equally to this work.

${ }^{2}$ Department of Bioinformatics and Biotechnology, Government College University Faisalabad (GCUF), Faisalabad, Pakistan

${ }^{1}$ College of Life Science and Technology, Guangxi University, Nanning, P. R.

China

Full list of author information is available at the end of the article
}

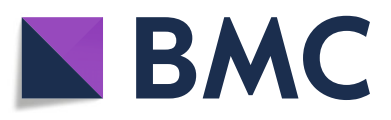

(- The Author(s). 2020 Open Access This article is licensed under a Creative Commons Attribution 4.0 International License, which permits use, sharing, adaptation, distribution and reproduction in any medium or format, as long as you give appropriate credit to the original author(s) and the source, provide a link to the Creative Commons licence, and indicate if changes were made. The images or other third party material in this article are included in the article's Creative Commons licence, unless indicated otherwise in a credit line to the material. If material is not included in the article's Creative Commons licence and your intended use is not permitted by statutory regulation or exceeds the permitted use, you will need to obtain permission directly from the copyright holder. To view a copy of this licence, visit http://creativecommons.org/licenses/by/4.0/ The Creative Commons Public Domain Dedication waiver (http://creativecommons.org/publicdomain/zero/1.0/) applies to the data made available in this article, unless otherwise stated in a credit line to the data. 


\section{Background}

Viruses have the potential to become dangerous life threat and cause irreparable loss to human beings. Hardly the world learns to cope with one strain of virus when another emerges and poses a threat to the future of humanity. A similar situation has emerged when a new strain of novel coronavirus $(\mathrm{CoV})$ that has not been previously identified in humans reported in December, 2019 [1, 2]. Coronaviruses are the largest among RNA viruses belonging to Coronaviridae, Roniviridae and Arteriviridae families. Coronaviridae are unsegmented, $3^{\prime}$ polyadenylated and $5^{\prime}$ capped positive sense singlestranded RNA viruses cause various respiratory diseases in humans $[2,3]$. CoVs are classified into four classes: alpha, beta, delta, and gamma. Amongst them, beta and alpha CoVs have been reported for infecting humans [4]. Recent $\mathrm{CoV}$ strain has received tremendous attention from researchers, as it causes a global pandemic of coronavirus disease 2019 (COVID-19) [5]. Severe acute respiratory syndrome coronavirus 2 (SARS-CoV-2) was identified as the causative agent of this pandemic [6]. The study of genome sequences has cast a shadow that SARS-CoV-2 is closely related to the SARS-CoV which is the causative agent of the SARS disease in 2002/2003 [7]. Initial diagnostic procedures indicated that the SARS-CoV-2 is primarily spread through respiratory droplets from sneezing/coughing, body contact and to some extent through fecal contact [8]. The SARS-CoV-2 may show symptoms within 14 days after exposure, or in some cases it takes more than 14 days. Symptoms of patients infected with COVID-19 include fever, runny nose, cough, and dyspnea [9]. Although the entire genome sequence of the virus has been published, the origin and proliferation mechanism of the new coronavirus is still ambiguous as stated by the World Health Organization [10]. Initial reports claimed that bats, snakes, pangolins or civet could be a possible animal source, but the claims are under debate and needs substantial research to prove it $[6,11-13]$. Researchers are currently working to sort out the SARS-CoV-2 source, including possible intermediate animal vectors.

The samples taken from a respiratory system-throat swab or lung fluid are helpful in diagnosing its infection in patients [14]. A special clinical diagnostic reverse transcription-PCR based test was developed [15]. Over 200 clinical trials are currently underway to test new and repurposed compounds against SARS-CoV-2 [16, 17]. Several medications such as hydroxychloroquine, remedesivir, and dexamethasone are being tested in clinical trials [18-21]. Several vaccines including subunit vaccines $[18,22]$, nanoparticle based vaccines, viral vector vaccines (adenovirus vector, Ankara vector), inactivated vaccines, fusion-protein based vaccines, recombinant protein, DNA vaccines, and live-attenuated vaccines are also being developed and in pre-clinical trials, but these vaccines are long months away from the market [23-27].

Immunoinformatics approaches can be applied to examine viral antigens, prediction of its epitopes and assessment of its immunogenicity [28, 29]. Moreover, this approach could be both time and cost-effective [3, 30, 31]. Excessive respiratory infection can also resolve with T-cell reactions and antibodies [32]. Furthermore, rapid identification, isolation, disease prevention, and control measures are required to hinder its spread of SARS-CoV-2 at homes, communities and healthcare units [33, 34]. In various studies, therapeutic approaches against the Ebola virus, Zika virus and Middle East respiratory syndrome corona virus (MERS-CoV) were developed using immunoinformatics approaches [3, 31, 35]. The purpose of this study was to pinpoint the potential T-cell and B-cell epitopes from SARS-CoV-2 structural proteins which can be further joined through adjuvant and linkers to design a multiepitope-based subunit vaccine (MESV). Many in silico approaches were used to validate the structural and physiochemical properties of the MESV. To examine the binding interaction and stability of MESV with human pathogenic receptors, molecular docking analysis has also been carried out. In addition, in silico immune simulation was also performed to validate the immunogenic potential of designed MESV. At the end, the MESV codons were optimized for Escherichia coli system and in silico cloning was performed to ensure its expression profiling. Flow chart of methodology used in present study is graphically presented in Fig. 1.

\section{Methods}

\section{Target proteins sequence and structural analyses}

Main structural proteins, Surface glycoprotein (S [Genbank: QHD43416.1]), Envelope protein (E [Genbank: QHD43418.1]) and Membrane glycoprotein (M [QHD43419.1]) of SARS-CoV-2 were taken as targets for epitopes screening and vaccine designing against SARSCoV-2. Their amino acid sequences were collected in fasta format from GenBank (https://www.ncbi.nlm.nih.gov/genbank/) [36]. Allergenicity and antigenicity (at a threshold of 0.4 ) of selected proteins were evaluated through AllerTOP v2.0 (https://www.ddg-pharmfac.net/AllerTOP/) and VaxiJen v2.0 (http://www.ddg-pharmfac.net/vaxijen/VaxiJen/VaxiJen.html) respectively [37, 38]. Three dimensional (3D) structure of S protein was retrieved from RCSB Protein Data Bank (PDB; https://www.rcsb.org/) [39]. However, 3D structures of other two proteins ( $E$ and $M)$ were predicted using homology modeling approach, as their resolved structures are not available yet. RaptorX (http:// raptorx.uchicago.edu/) and MODELLER v.9.12 (https:// salilab.org/modeller/) were employed for homology modeling [40]. Predicted models were then visualized by Chimera (https://www.cgl.ucsf.edu/chimera/) [41]. Galaxy 


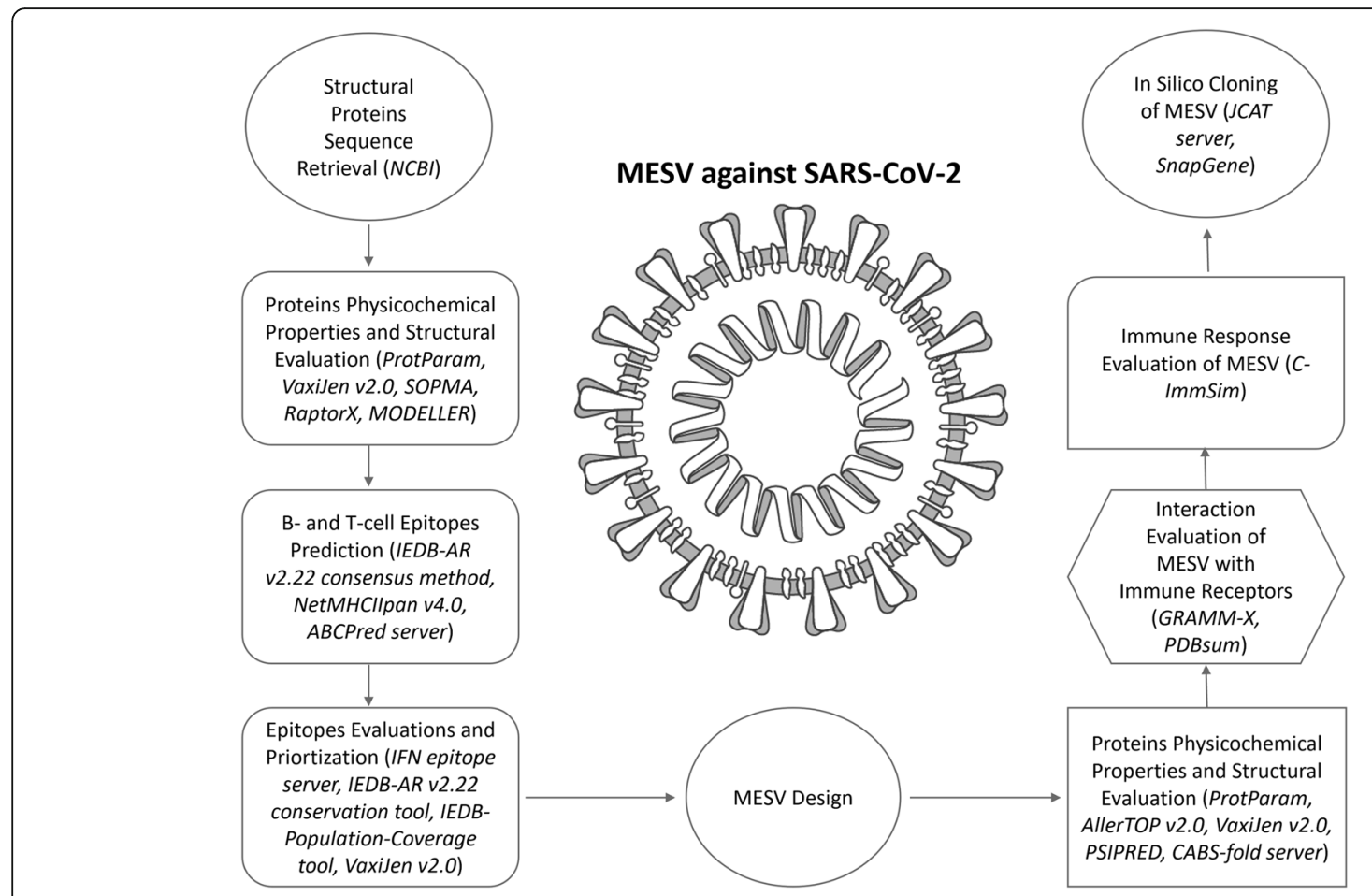

Fig. 1 The schematic workflow used to develop MESV construct against SARS-CoV-2 structural proteins

refines server (http://galaxy.seoklab.org/) and ModRefiner (https://zhanglab.ccmb.med.umich.edu/ModRefiner/) was used to refine the predicted models [42, 43]. Besides, the refined structure needs to be validated based on experimentally validated 3D structure of proteins. Refined structures were therefore applied in the PROSA web (https:// prosa.services.came.sbg.ac.at/prosa.php) providing a quality score for a given structure [44]. The quality score beyond the usual range of native proteins indicates a possible error in protein structure. Ramachandran plot was created by rampage server (http://mordred.bioc.cam. ac.uk/ rapper/rampage.php), where the principle of PROCHECK is applied to validate the protein structure [45]. Structural analysis was performed to later investigate the positions of B-cell epitopes on target proteins.

\section{Prediction of B-cell and T-cell epitopes}

The epitopes of B-cells help to detect viral infections in the immune system. ABCpred (http://crdd.osdd.net/ raghava/abcpred/) was used to forecast 14-mer B cell epitopes for target proteins at 0.51 threshold [46]. Epitopes evident on the outer surface were picked, and other intracellular epitopes were removed. The Vaxijen server tested the antigenicity of the selected epitopes at a threshold of 0.5. B-cell epitope identification was based upon antigenicity, flexibility, linear epitope predictions, hydrophilicity, and surface accessibility [47]. Parker hydrophilicity prediction algorithms, Emini surface accessibility prediction method, Kolaskar and Tongaonkar antigenicity scale, and Karplus and Schulz flexibility prediction tool were used to perform hydrophilicity, accessibility of surface, antigenicity and flexibility analysis respectively [48]. As discontinuous epitopes become more evident and have higher dominant properties than linear epitopes, DiscoTop 2.0 server (http://www.cbs.dtu. $\mathrm{dk} /$ services/DiscoTope/) was used to forecast discontinuous epitopes from 3D structures of surface glycoprotein, membrane protein and envelope protein [49]. The position of epitopes on 3D structures of proteins was visualized by Pymol (https://pymol.org/2/) [50].

In vaccine designing, $\mathrm{T}$-cell epitopes play a crucial role. More specifically, it reduces the cost and time compared with laboratory experiments [51]. IEDB consensus method (http://tools.iedb.org/mhcii/) was used to predict $8-11$ mer MHC class-I and 11-14 mer MHC class-II epitopes. The results of this method are very important due to a large number of human leukocyte antigen (HLA) alleles used in the calculation. The sequence was given in a FASTA format and all the alleles were selected for prediction. Epitopes with less than 2 consensus score believed to be good binders and chosen for further research.

\section{Evaluation of predicted epitopes}

Antigenicity and allergenicity of the selected epitopes were checked by Vaxijen v2.0 and Allergen FP v1.0 respectively [52]. Protein Digest server (http://db.systemsbiology.net: 
8080/proteomicsToolkit/proteinDigest.html) was used to predict epitopes digesting enzymes. ToxinPred (http:// crdd.osdd.net/raghava/toxinpred/) was used for nontoxic/toxic properties prediction of epitopes. Non-toxic epitopes were selected for further analysis [53].

\section{Epitopes conservation and population coverage analysis}

The degree of conservation of predicted $\mathrm{T}$-cell and B-cell epitopes within the protein sequence was analyzed by IEDB conservancy analysis tool (http://tools.iedb.org/conservancy/). Epitopes having conservancy among all 3 selected proteins were shortlisted for further analyses [54].

The expression and distribution of HLA alleles vary depending on the world's ethnicities and regions, thereby impacting the effective production of MESV [55]. The population coverage was calculated using the IEDB population coverage tool (http://tools.iedb.org/population/), and for this purpose MHC class-I and MHC class-II epitopes and corresponding HLA-binding alleles were considered. This tool estimates population coverage for each epitope for various regions of the world based on the distribution of HLA binding alleles [56].

\section{Multi-epitope-based subunit vaccine (MESV) designing and evaluation}

Epitopes with the following characteristics are generally preferred to design a subunit vaccine: (a) highly antigenic, (b) immunogenic, (c) non-allergenic, (d) non-toxic, and (e) with significant population coverage. Therefore, only those epitopes were selected further to construct MESV following the above parameters. An adjuvant was attached with the EAAAK linker to the first cytotoxic T lymphocytes (CTL) epitope to improve the immune response. Other epitopes were linked using AAY, GPGPG, and KK linkers after validation of their interaction compatibility to preserve their independent immunogenic activity. $\beta$ defensin has been used as an adjuvant in the present research since it is a simple 45 amino acids long peptide that acts as an immunomodulator and as an antimicrobial agent both [57].

First, Blastp analysis was carried out using default parameters to confirm that the designed MESV sequence is non-homologous against the Homo sapiens proteome [58]. Protein with less than $37 \%$ is commonly known to be a non-homologous. Physiochemical properties of the designed MESV were accessed by the Protparam tool [59]. Protparam predicts various physiochemical properties like (half-life, theoretical isoelectric point [pI], instability index, grand average hydropathy, and aliphatic index) based on the amino acid approximations involved in the pk [60]. AllerTOP v.2.0 server was used to analyze the allergenicity of the MESV construct [38]. The secondary structure of the MESV construct was evaluated using a PSIPRED workbench [58]. This test also evaluated various vaccine properties such as alpha helices, extended chain, degree of beta turns, and random coil.

The 3D structure of MESV was predicted using the de novo modeling approach of CABS fold server (http://biocomp.chem.uw.edu.pl/CABSfold/), since the designed MESV was a series of epitopes and no appropriate template was available [61]. This server is based on a CABS modeling approach that combines a multi-scale modeling pipeline with an exchange replica Monte Carlo scheme. Predicted MESV 3D structure was modified using a galaxy refine server [62]. The Ramachandran plot analysis was carried out using the RAMPAGE server (http://mordred. bioc.cam.ac.uk/ rapper/rampage.php) [45], to confirm the quality of the refined MESV structure, followed by the structural validation analysis using the PROSA web server [44]. The ERRAT server (https://servicesn.mbi.ucla.edu/ ERRAT/) was also used to evaluate the calculation of unbounded interactions in the MESV structure [63].

Besides, linear B-cell epitopes were predicted from the MESV using the ABCpred server [46]. Ellipro tool (http:// tools.iedb.org/ellipro/) was used to predict the conformational B-cell epitopes of the designed MESV using default settings (maximum distance: $6 \mathrm{~A}^{\circ}$; minimum score: 0.5 ), provided by IEDB-AR v.2.22. It predicts epitopes by estimating residual protrusion index (PI), protein shape, and neighbor residue clustering [64].

\section{Molecular docking of MESV with human immune receptors}

All together for the appropriate evocation of immune response, the interaction amongst the antigenic molecule and immune receptor molecule is essential. Molecular Docking was performed to analyze the interaction between MESV construct and human immune receptors. Toll-like receptors-3 (TLR3) has been thoroughly studied, and studies found its key role in antiviral immune response generation. GRAMM-X (http://vakser.compbio. $\mathrm{ku}$.edu/resources/gramm/grammx/) was used for the MESV docking with TLR3 (PDB ID: 1ZIW) [65]. Pymol was utilized for visualization of the docked complexes [50]. Moreover, for the achievement of the conventional sketch of interactions among docked proteins, an online server PDBsum (http://www.ebi.ac.uk/thornton-srv/databases/cgi-bin/pdbsum/GetPage.pl?pdbcode=index.html) was utilized. It analyzes the protein-protein interactions among docked molecules [66].

\section{Immunogenicity evaluation of the vaccine construct}

An in silico immune simulation was performed using CImmSim 10.1 server (http://150.146.2.1/C-IMMSIM/ index.php?page $=0$ ) to validate the immunological responses of the designed MESV. C-ImmSim simulates the three main components of the functional mammal system (Thymus, lymph node, and bone marrow) [67]. The input 
parameters for the immune simulations are as follows: volume (10), HLA (A0101, A0101, B0702, B0702, DRB1_ 0101, DRB1_0101), random seed (12345), number of steps (100), number of injection set to 1 . The rest of the parameters were considered to be the default.

\section{In silico cloning and codon optimization}

Codon optimization is a method to improve the translation effectiveness of foreign genes in the host if the use of codon is different in both organisms. Codon optimization was carried out followed by in silico cloning, after the careful evaluation of MESV properties and immune response. To make this method consistent with the commonly used prokaryotic expression system; E. coli K12 [68], the java codon adaptation tool (http://www.jcat.de/) [69] was used for MESV codon optimization. The other available choices were selected to evade: (i) termination of rho-independent transcription, (ii) binding-site of prokaryote ribosome, and (iii) cleavage-sites of restriction enzymes. Codon adaptation index (CAI) [70] along with the GC (guanine and cytosine) contents were assessed. Sticky ends of the restriction sites of HindIII and BamHI restriction enzymes were added to allow restriction and cloning, in the start/ $\mathrm{N}$ terminal and end/C terminal of the modified MESV sequence, respectively. The modified nucleotide sequence of MESV was additionally cloned into the E. coli pET30a (+) vector by using SnapGene tool (https://www.snapgene.com/ ), to assure its in vitro expression.

\section{Results}

\section{Sequence and structural analysis of the target proteins}

All target structural proteins were found to be nonallergenic and highly antigenic. E protein was the most antigenic followed by $M$ and $S$ protein with $0.60,0.51$ and 0.46 antigenic values, respectively.

The 3D structure of $\mathrm{S}$ protein was retrieved from Protein-Data-Bank using ID: 6VYB [39]. The 3D structure of $\mathrm{E}$ protein was determined using homology modeling. Chain-A of envelope small membrane protein of SARSCoV (PDB ID: 5X29) was found to be the best template (percent identity $88.71 \%$ ) for E protein of SARS-CoV-2. However, no suitable template was found for M protein, so its structure was predicted by Raptor X [71]. Visualization of the models was done by Chimera (Additional file 1: Fig. S1). The quality factor (z-score) and Ramachandran plot values of refined predicted models are mentioned in Additional file 2: Table S1 (Additional file 1: Fig. S2-S3).

\section{B-cell epitopes prediction from target proteins}

Total 23 linear epitopes (S-19, E-1, and M-3) were selected. Among the chosen linear epitopes, 'ILPVSMTKTS VDCT' of S protein showed the highest antigenicity (1.6) and predicted score (Additional file 3: Table S2). The positions of epitopes on their respective protein structures were visualized by Pymol (Additional file 1: Fig. S4).

Identification of B cell epitope was based on antigenicity, flexibility, linear epitope predictions, hydrophilicity, and surface accessibility. Parker hydrophilicity prediction algorithms, Emini surface accessibility prediction method, Kolaskar and Tongaonkar antigenicity scale, and Karplus and Schulz flexibility prediction tool were used to perform hydrophilicity, accessibility of surface, antigenicity and flexibility analysis respectively (Additional file 1: Fig. S5-S7).

To further improve the specificity and variety of B-cell epitopes, Discotop 2.0 server was used to calculate surface abundance concerning residual contact number and use the novel amino acid score to forecast discontinuous epitopes. 3D structures of the target proteins were used to predict discontinuous epitopes; $90 \%$ specificity, -3.700 thresholds and 22.000 Angstroms propensity score radius. Fifty-five discontinuous epitopes of $S$ protein, 1 epitope of the E protein and 22 epitopes of $\mathrm{M}$ protein were calculated (Additional file 5: Table S4).

\section{T-cell epitopes prediction from target proteins}

Epitopes that are bound to multiple alleles, highly antigenic, non-allergenic and 100\% conserved were screened out, and their antigenicity and allergenicity were checked. Based on these criteria, 9 MHC class-I (S-3, E-3, and M-3) and 7 MHC class-II (S-1, E-3 and M-3) were shortlisted (Additional file 6: Table S5). Protein Digest server was used to estimate epitopes/peptides digesting enzymes. Epitopes digestible with many enzymes are not stable. Less enzyme digested epitopes, on the other hand, are very stable and favored vaccine candidates (Additional file 7: Table S6).

\section{Evaluation and selection of epitopes for further analyses}

Total three CTL epitopes (S-1 and M-2), six HTL epitopes (E-3 and M-3), and four B-cell epitopes (S-3 and M-1) were selected to construct MESV (Table 1).

The selected epitopes showed $88.40 \%$ of the world population coverage (Fig. 2). Results revealed that predicted epitopes are showing promising population coverage of the countries strongly affected by COVID-19 including, Germany, France, Spain, Saudi Arabia, England, Italy, Iran, the Philippines, the United States, and Sweden.

\section{Designing of MESV}

A MESV construct was further developed using all selected epitopes. Using the EAAAK linker, an adjuvant (45 amino acid long B-defensin) was bound at the beginning (to the MEV N-terminal). EAAAK linker reduces connections to other protein areas with efficient detachment and improves stability [58, 72]. Epitopes were merged in a sequential manner with AAY, GPGPG, and KK linkers, respectively, based on the compatibility of their interaction. Two hundred seventy-six amino acids represented the final MESV construct (Fig. 3). 
Table 1 Final selected epitopes from SARS-CoV-2 structural proteins used to design the multi-epitope-based subunit vaccine (MESV) construct

\begin{tabular}{|c|c|c|c|c|c|c|}
\hline Sr.No & Epitope & Protein & Position & HLA alleles & Antigenicity & Immunogenicity \\
\hline \multicolumn{7}{|c|}{ MHC class-I } \\
\hline 1 & VRFPNITNLCPF & S & $327-338$ & HLA-B*35:01 & 1.2 & 0.11 \\
\hline 2 & YRINWITGGIAI & M & $71-82$ & HLA-B*27:05 & 1.2 & 0.61 \\
\hline 3 & SFRLFARTRSMW & M & $99-110$ & HLA-B*57:01 & 0.6 & 0.05 \\
\hline \multicolumn{7}{|c|}{ MHC class-II } \\
\hline 1 & LLFLAFWFLLVTLA & $E$ & $18-32$ & HLA-DRB 1*04:04 & 0.8 & 0.41 \\
\hline 2 & AFWFLLVTLAILTA & E & $22-36$ & HLA-DRB1*04:01 & 0.6 & 0.39 \\
\hline 3 & FWFLLVTLAILTAL & E & $23-37$ & HLA-DRB1*04:01 & 0.5 & 0.38 \\
\hline 4 & VTLACFVLAAVYRIN & M & $60-74$ & HLA-DRB 1*04:08 & 1.0 & 0.36 \\
\hline 5 & ASFRLFARTRSMWSF & M & $98-112$ & HLA-DRB1*04:01 & 0.7 & 0.13 \\
\hline 6 & FRLFARTRSMWSFNP & M & $100-114$ & HLA-DRB1*04:01 & 0.8 & 0.11 \\
\hline \multicolumn{7}{|l|}{ B-Cell } \\
\hline 1 & SPTKLNDLCFTNVY & S & 383 & - & 1.6 & 0.67 \\
\hline 2 & EILDITPCSFGGVS & S & 583 & - & 1.6 & 0.81 \\
\hline 3 & ILPVSMTKTSVDCT & S & 726 & - & 1.6 & 0.89 \\
\hline 4 & LEQWNLVIGFLFLT & M & 17 & - & 0.9 & 0.67 \\
\hline
\end{tabular}

\section{Sequence and structural analyses of MESV}

First, Blastp analysis was carried out against the Homo sapiens proteome, and the results revealed that MESV does not resemble any human protein (higher or equal to $37 \%)$. The vaccine structure was then tested for toxicity, allergenicity, and antigenicity. MESV was found to be non-allergenic, highly antigenic (0.6737), and non- toxic. The mean half-life of the construct was calculated as $30 \mathrm{~h}$ in vitro, $>20 \mathrm{~h}$ in yeast and $>10 \mathrm{~h}$ in vivo. Molecular weight and theoretical pI of the vaccine were $3157.01 \mathrm{kDa}$ and 10.31 respectively. Grand average hydropathicity was calculated as 0.395 . A positive score of the grand average of hydropathy suggests its hydrophobic nature. The secondary structure analysis show

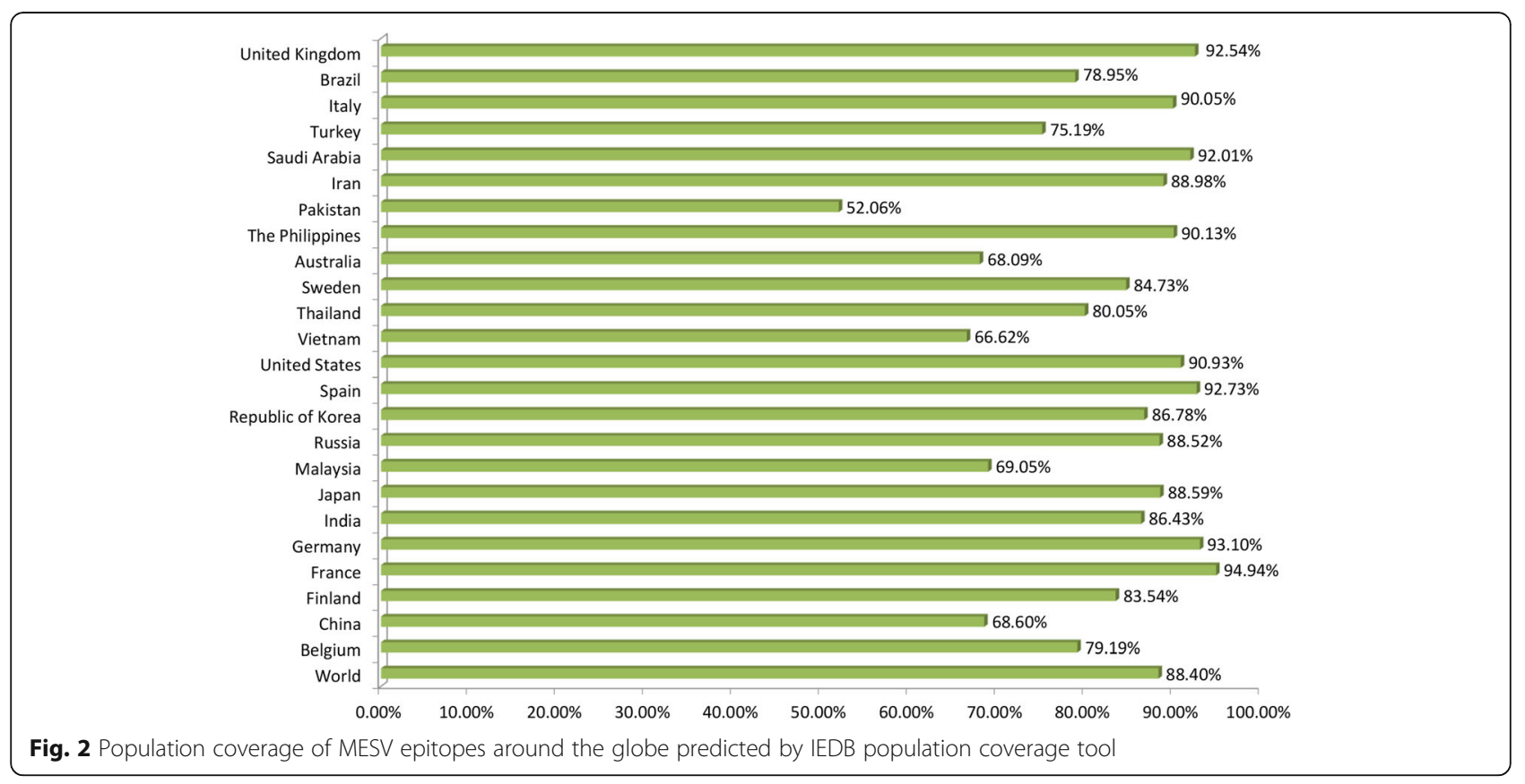




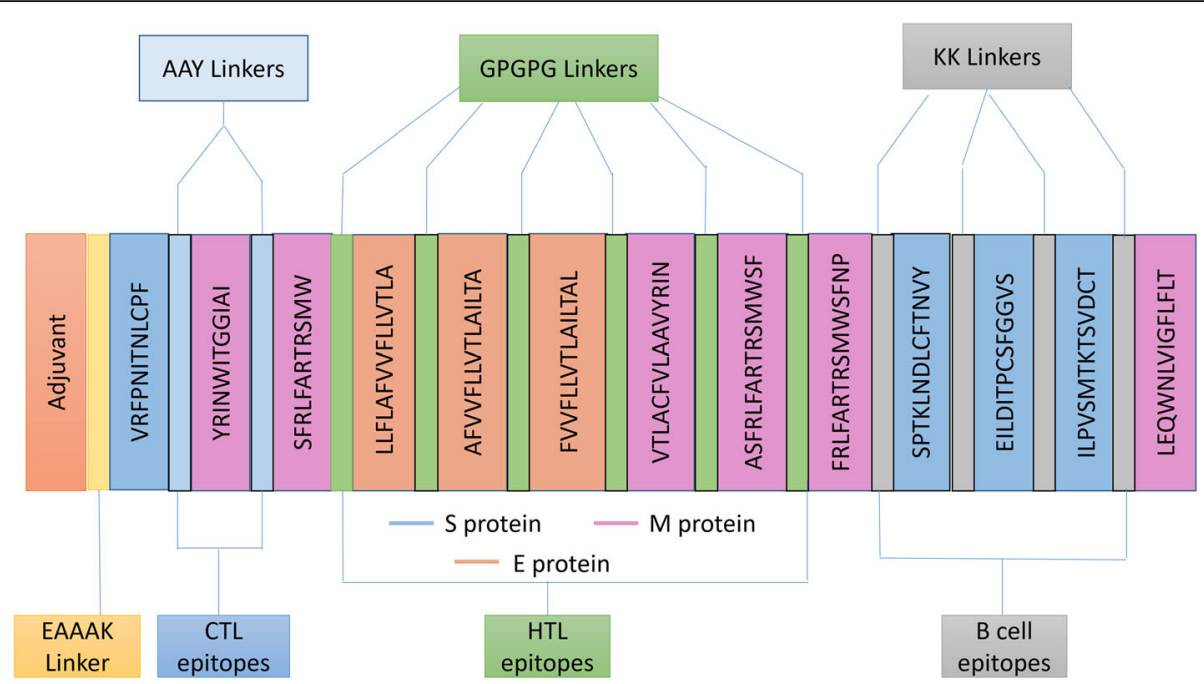

Fig. 3 Schematic diagram of MESV construct: It has 276 amino acids, consisting of an adjuvant (orange) linked at N-terminal of MEV with the aid of EAAAK linker (yellow). AAY linkers (blue) used to join the CTL epitopes, GPGPG linkers (green) used to join the HTL epitopes and KK linkers (gray) used to join the B-cell epitopes

that $74(26.81 \%)$ amino acids were involved in the formation of $\alpha$-helix, 67 amino acids (24.27\%) formed $\beta$ strands and 135 amino acids (48.91\%) form coils.

CABS fold server was used to predict the tertiary structure of the MESV (Fig. 4). The structure was refined by the galaxy refine server. Ramachandran plot analysis of improved model showed that $89.4 \%$ amino acids are in favored region, $6.9 \%$ amino acids in the allowed region and $3.6 \%$ amino acids in the outlier region. Further analysis showed that the qRMSD is 0.544 , MolProbity is 2.356 , poor rotamers are $0.0 \%$, clash score is 17.7 and $\mathrm{z}$-score is -4.8. In quality check analysis by ERRAT, the refined model score was 82.4561 .

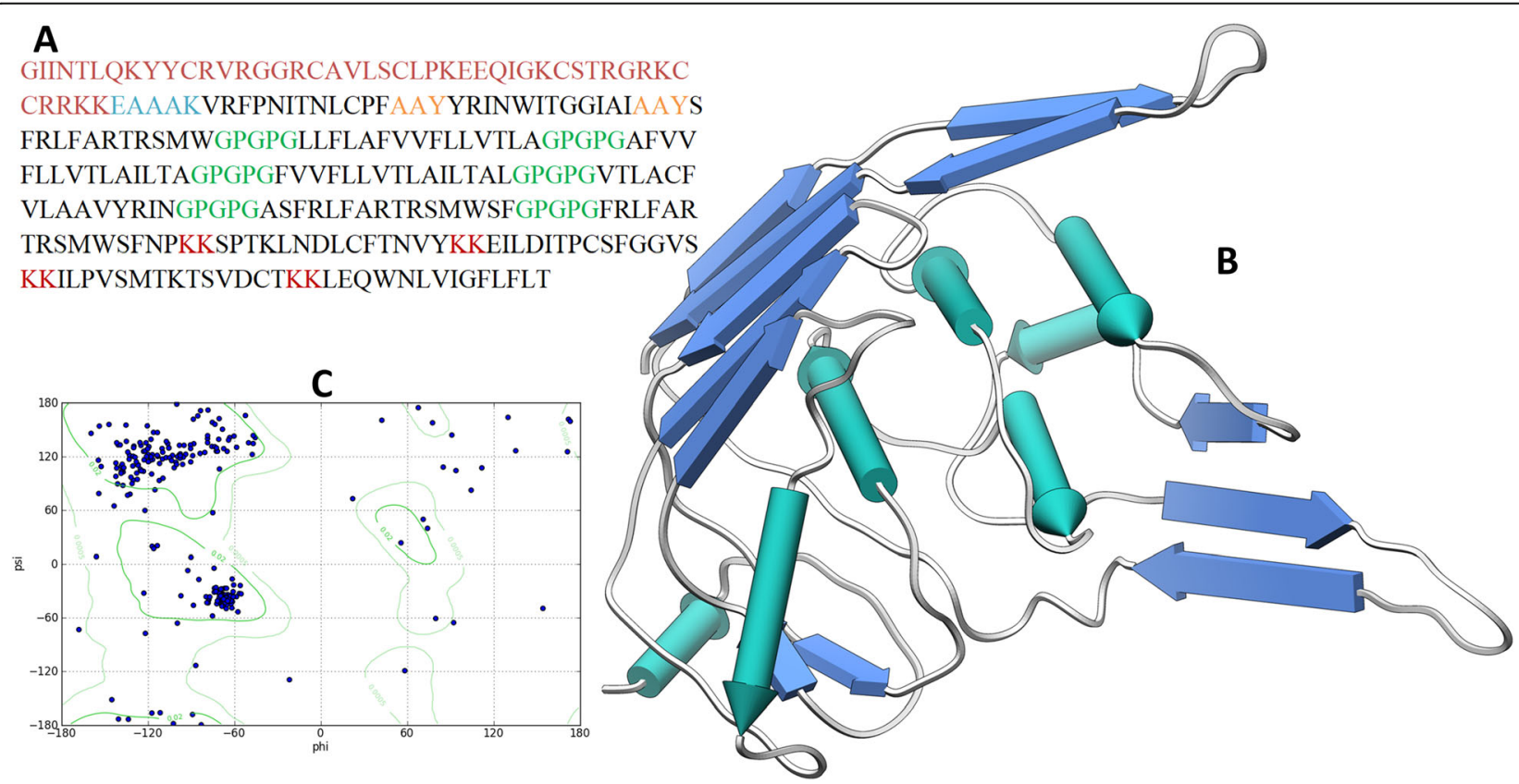

Fig. 4 a MESV construct sequence. Epitopes sequence is in black. The adjuvant sequence is highlighted in brown color, EAAAK linker sequence is highlighted in blue, AAY linkers are highlighted with orange, GPGPG linkers are highlighted with green and KK linkers are highlighted with maroon color; b MESV construct refined 3D structure pipes representation (alpha helix: green; beta strands: blue; loops: gray); c Ramachandran plot analysis of predicted structure shows $89.4 \%$ residues are present in the favored region 


\section{B-cell epitopes screening from MESV}

B-lymphocytes also produce antibodies that provide humoral immunity, in addition to the secretion of cytokines. Eighteen linear/continuous (Additional file 8: Table S7) and six conformational/ discontinuous epitopes (Additional file 9: Table S8) from the MESV sequence were predicted without altering ABCPred 2.0 and Ellipro prediction parameters.

\section{Molecular docking of MESV construct with TLR3}

To start the immune response, an appropriate interaction among the antigenic molecule and immune receptor molecule is needed. To decode the binding potential of MESV to the innate immune receptors, bioinformatic modeling driven molecular docking of the designed MESV to a representative innate immune receptor TLR3 was performed. The docking evaluation forecast that the best complex with a net global energy of $-22.36 \mathrm{~kJ} / \mathrm{mol}$. Visual analysis of the complex leads to the observation of the MESV's deep binding in the center of TLR3 and favors rigorously hydrogen and weak van dar Waals interactions with specific TLR3 residues. PDBsum was used to gain insights and pin down possible residues of MESV making stable bonds with TLR3 (Fig. 5). Within $3 \AA$, the MESV was observed to form 14 hydrogen bonds with TLR3 potential residues.

\section{Immunogenicity evaluation of MESV}

All secondary and primary immune responses tend to contribute significantly to the pathogen and may be consistent with the actual immune response. The in silico host immune system response to the antigen is shown in Fig. 6. The primary response was characterized by high IgG + IgG and IgM concentration, followed by IgM, $\operatorname{IgG} 1+\operatorname{IgG} 2$ and IgG1 at both the secondary and primary stages with concomitant antigen reduction. Additionally, robust interleukin and cytokine response was observed. All of this indicates the MESV's successful immune response and clearance after subsequent encounters.

\section{In silico cloning within E. coli system}

In silico cloning was done to assure the expression of MESV derived from SARS-CoV-2 in widely used E. coli hosts. First, the codons of MESV were modified according to the use of codons of E. coli expression system (strain K12). The optimized MESV construct contains 828 nucleotides, CAI value of $1.0(0.8-1.0)$, and an optimal range of GC content of $53.2 \%(30-70 \%)$ demonstrating the strong potential for reliability and positive protein expression. In the following step, both ends of MESV optimized nucleotide sequence were attached to buffer compatible restriction enzymes BamHI and HindIII restriction sites to assist the purification/cloning process. Finally, the refined MESV sequence was cloned to the several cloning sites of the pET30a (+) vector between the restriction sites. The clone was $6.23 \mathrm{~kb}$ long (Fig. 7).

\section{Discussion}

CoVs have long been considered as insignificant pathogens causing "colds" in humans. In the twenty-first century, two extremely pathogenic CoVs named SARS-CoV and MERS-CoV emerged from the livestock reservoirs and cause deadly outbreaks. A new strain of CoV officially named as SARS-COV-2 was identified recently, which started a deadly global pandemic of COVID-19. The final dimension and impact of this pandemic are currently uncertain due to the rapidly changing situation [4]. After the recombination of various virus genomes particles, the novel virus infects the host cells rapidly. No reliable medication is currently available for the said infection. COVID-19 infection is a severe problem of morbidity and mortality worldwide. Unfortunately, the unavailability of the vaccinations against COVID-19 has impacted several precious lives, in different regions of the world. The emergence of COVID-19 results in a significant global disease burden, for which preventative measures are urgently needed. To successfully eradicate the disease, researchers have been trying to collect data associated with CoVs to understand its transmission, pathophysiology, and biology [73]. The rapid development of structural and genomic databases combined with computational tools helps in the design and discovery of new vaccine candidates.

Recent advancements in the immunological bioinformatics area have resulted in a variety of tools and servers that can lessen the time and cost of traditional vaccine advancement. Due to the problems in the selection of suitable antigen candidates and immunodominant epitopes, the development of effective multiepitope vaccines remains toilsome. Thus, the prediction of appropriate antigenic epitopes of a targeted protein by the immunoinformatics approaches is very essential for designing a MESV [74].

Here, we explored the development of epitope-based vaccines targeting the structural proteins $(\mathrm{S}, \mathrm{M}$, and $\mathrm{E})$ of the SARS-CoV-2. These proteins play a crucial in the replication cycle and the virus particle structure. The Sprotein plays an important part in binding the virus to the host cell surface receptors and consecutive fusion to promote the viral entrance in the host cell [75-77]. M and $\mathrm{E}$ proteins are important for replication, particle assembly within human cells, and viral entry [78, 79]. Tand B-cell epitopes of the target proteins were predicted to support the host's immune response. The research was performed at primary, secondary and tertiary structural levels of proteins. IEDB analysis resource and ABCPred predicted B-cell conserved epitopes. The position of epitopes on 3D structures of proteins was visualized by Pymol. DiscoTop server was used to predict 


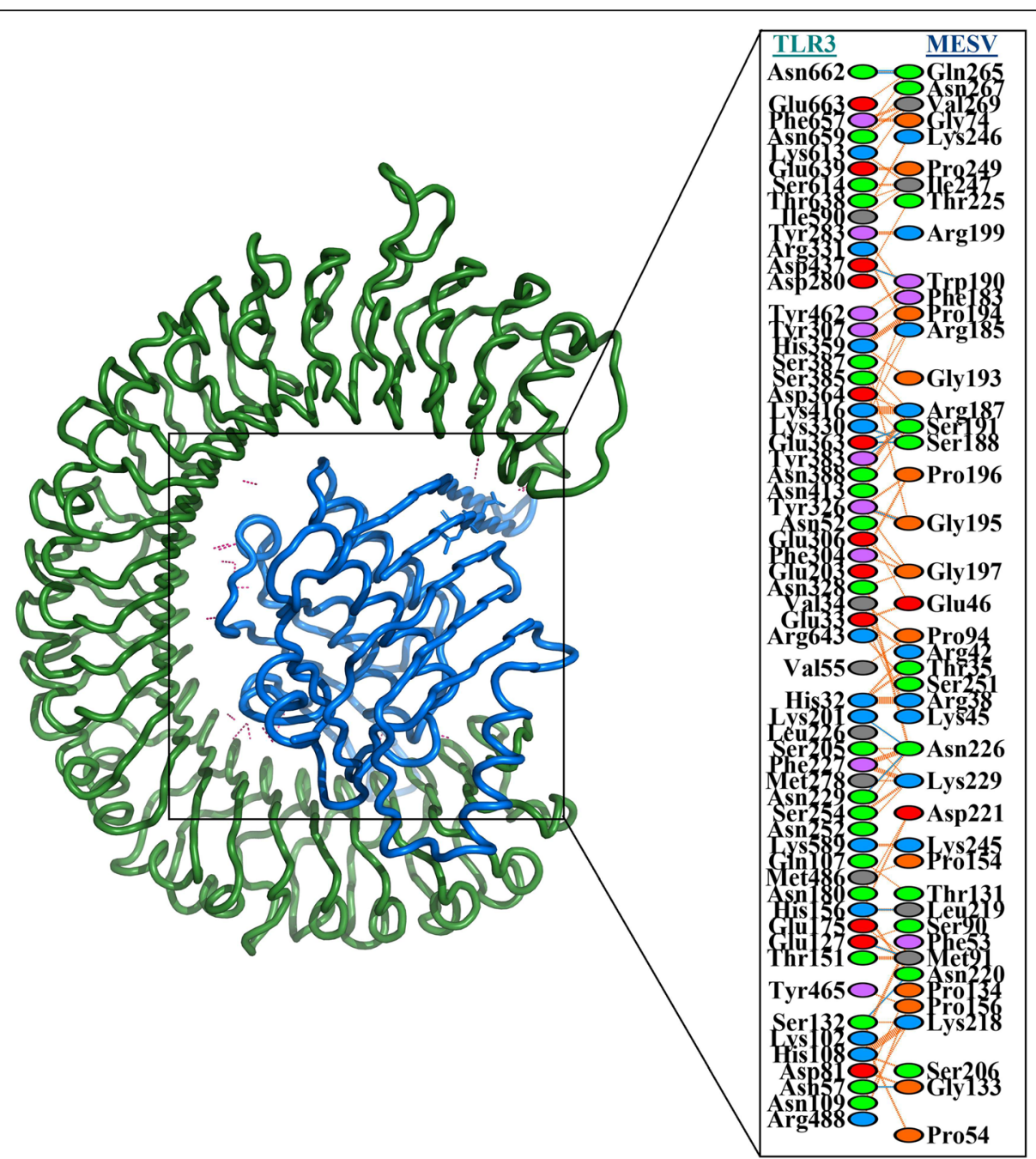

Fig. 5 TLR3-MESV docked complex shown at the left in cartoon representation. Interacting residues of MESV are highlighted at right side. MESV vaccine construct displayed with blue color and TLR3 displayed with green color. Salt bridges are displayed with red color lines; other contacts are shown with orange color lines, and hydrogen bonds are displayed with blue color lines. The colors of interacting residues are interpreting the characteristics of amino acids (neutral: green, Cys: yellow, aromatic: pink, aliphatic: grey, positive: blue, negative: red, and Pro\&Gly: orange)

discontinuous epitopes. To further improve specificity and selectivity, allergenicity, toxicity, and physiochemical properties of predicted epitopes were checked. Digestion analysis verified that the peptides predicted during the analysis were stable and safe to use.

An appropriate MESV should be designed with B-cell, HTL, and CTL epitopes and cause effective reactions to a specific virus [80]. Few groups developed SARS-CoV-2 subunit vaccines but only used a single protein for the vaccine design $[15,81,82]$ and the use of CTL epitopes only without taking into account the importance of HTL or B cell epitopes [83]. However, we have incorporated $\mathrm{B}$-cell epitopes in addition to $\mathrm{T}$-cell epitopes from multiple structural proteins, because of the functions they play in inducing antibody production and mediating its effective features [84]. Besides, the humoral response of memory B-cells can be easily overcome by the onset of antigens, while the cell-mediated immunity (T-cell immunity) in many cases leads to long-life immunity [85]. CTL limits pathogen spread through the secretion of unique antiviral cytokines and the identification and destruction of infected cells [86]. Therefore, the present vaccine construct has an advantage over already reported constructs.

The HLA alleles retain their response to T-cell epitopes which are highly polymorphic in different ethnic groups. To gain more population coverage, the T-cell epitopes are paired with more alleles. So we chose the HTL and CTL epitopes with their respective HLA alleles to predict the worldwide distribution of the alleles. The results showed that the chosen epitopes and their corresponding alleles preferably cover various geographical 

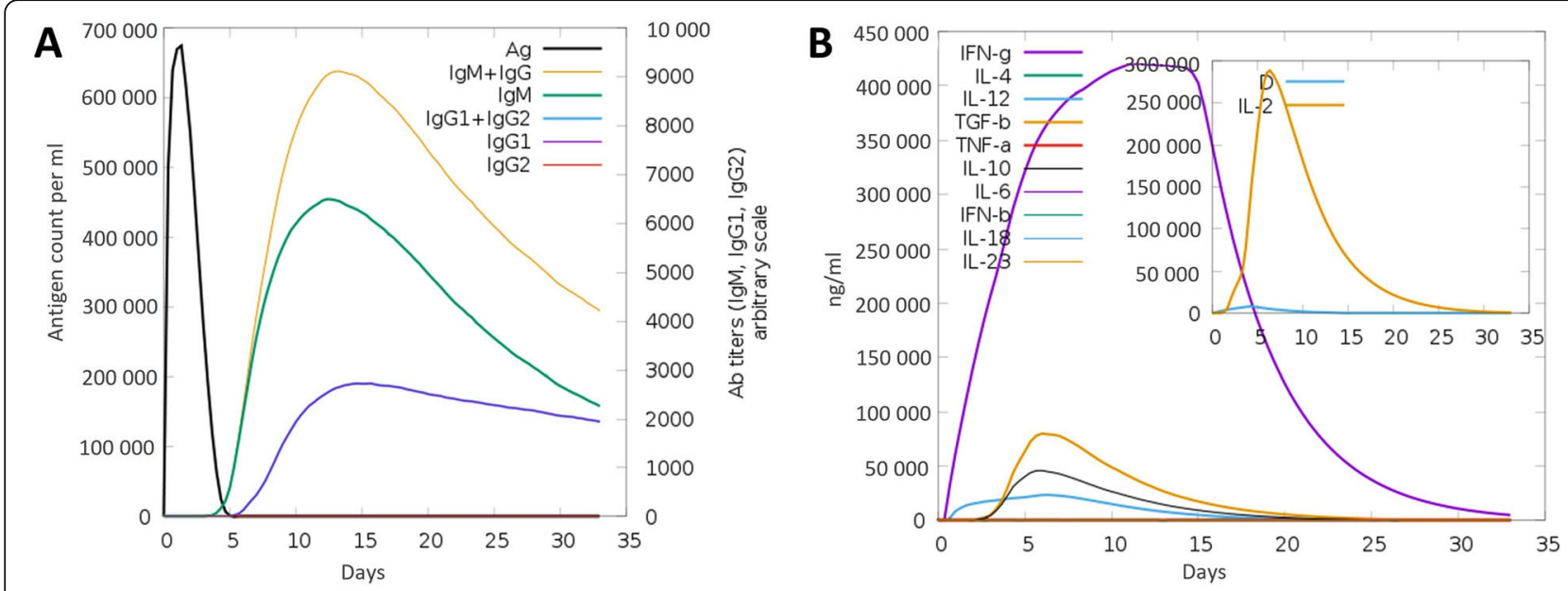

Fig. 6 In silico immune response using MESV as antigen. a The antibodies, and $\mathbf{b}$ cytokines and interleukins

areas of the world. The selected epitopes cover $88.40 \%$ of the world population. France has the highest population, with 94.94\%. In Germany, Spain, Saudi Arab, England, and Iran, the epidemic of SARS-CoV-2 happened in most significant measures. Vaccine candidates are therefore vital to protect individuals from SARS-CoV-2 infection in these geographical regions. The population coverage was $68.60 \%$ in China, where the virus first emerged and had several outbreaks.

Vaccine candidates were chosen form CTL, HTL, and B cell epitopes depending on their antigenicity, toxicity, immunogenicity, population coverage, and allergenicity.

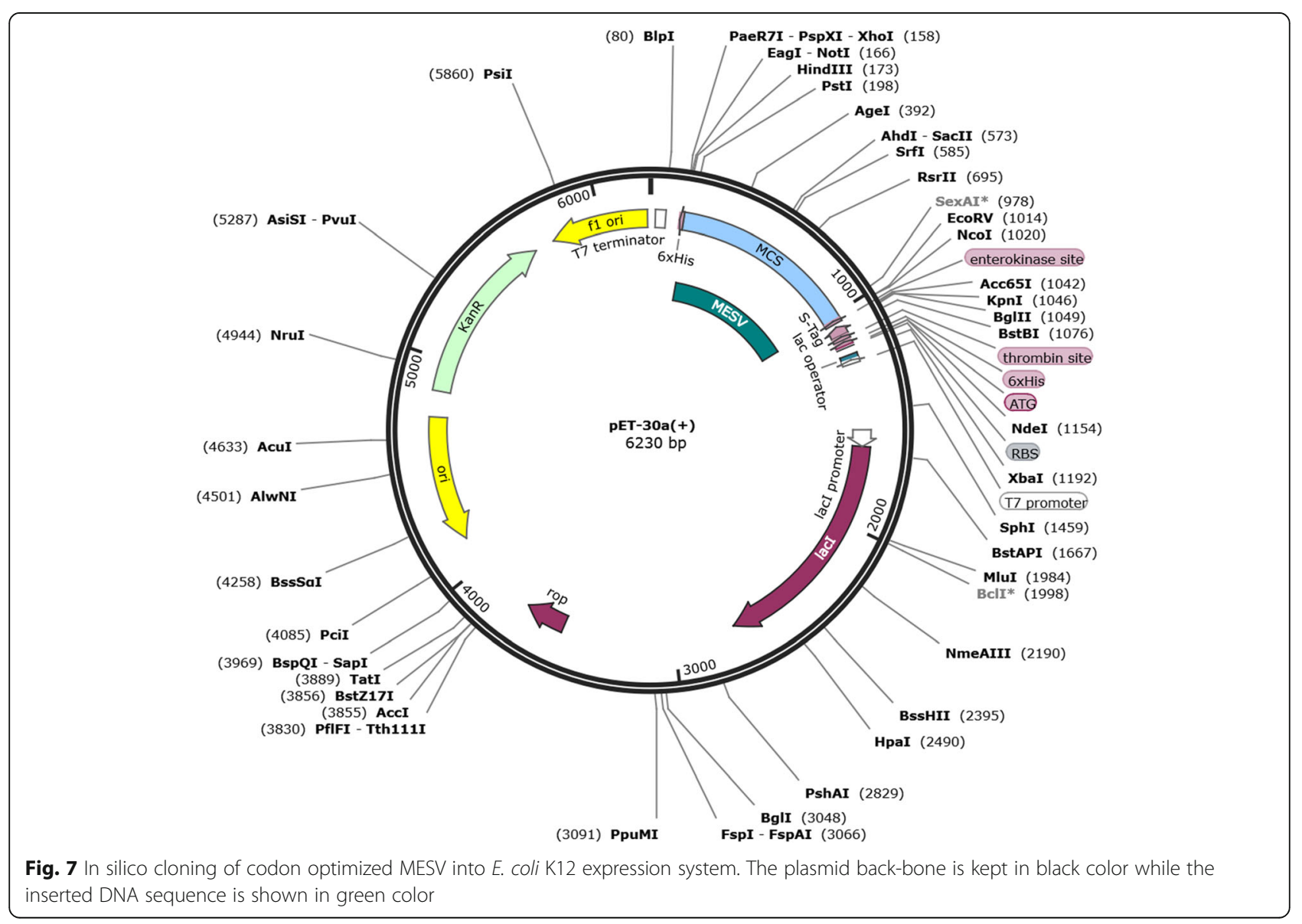


The MESV was designed by joining the HTL, CTL, and B cell epitopes with GPGPG, AAY, and KK linkers respectively. Linkers are introduced as an indispensable element in the MESV development to enhance folding, stabilization, and expression. Multi-epitope based vaccines are poorly immunogenic when used alone, and need adjuvant coupling [87]. Adjuvants are ingredients in a vaccine formulation that protects against infection and affect certain immune responses, growth, stability, and durability of antigens [88]. Therefore, 45 amino acids long, an adjuvant $\beta$-defensin, was integrated with the EAAAK linker whose length is 5 , at $\mathrm{N}$-terminal. The EAAAK linker is used to integrate the first epitope and adjuvant to facilitate efficient separation of the bifunctional fusion protein domains [89]. The final vaccine stretch with the addition of adjuvant and linkers was discovered to be 276 amino acid long.

The analysis of physiochemical characteristics of the MESV construct has shown that it is stable, basic, and hydrophobic. MESV was basic, according to the theoretical $\mathrm{pI}$ value, which can ensure stable physiological $\mathrm{pH}$ interaction. The calculated aliphatic index and instability index scores showed that the vaccine protein may be stable and thermostable. A positive score of the grand average of hydropathy suggests its hydrophobic nature. MESV has been found to be immunogenic, strongly antigenic, and non-allergenic. This suggests the ability of the epitopic vaccine to elicit a strong immune response without allergic reactions.

The 3D structure prediction provides extensive knowledge of the spatial arrangement of essential protein components and provides excellent support for the study of ligand interactions, protein functions, dynamics, and other proteins [90, 91]. After refinement, the desirable characteristics of the MESV construct improved considerably. The Ramachandran plot analysis shows that most residues are present in favored and allowed regions with very few residues in the disallowed region, which shows a satisfactory overall quality of the model. The good quality of designed MESV construct is further indicated by RMSD value, Poor Rotamers, Clash Score, and MolProbity. Various structure validation methods have been used to detect errors in the modeled MESV construct. The ERRAT quality factor $(82.4 \%)$ and $\mathrm{z}$-score $(-4.8)$ proved that the overall structure of the refined MESV is of good quality.

An adequate interaction between the antigen molecule and the immune receptor molecules is important for triggering an immune response. The refined MESV construct was then docked against TLR3 to examine adequate binding to immediate immune response. Stable interactions were observed among the MESV and TLR3 in molecular docking analysis, and less energy was needed for proficient binding.
B- and T-cell epitopes consisting multi-epitope vaccine should hypothetically activate both humoral and cellular immune reactions. With substantial IL-10 and IL-2 activities, our vaccine demonstrated the highest production of IFN- $\gamma$. Antibodies also provide extracellular SARS$\mathrm{CoV}-2$ protection. We have also noticed excess immunoglobulins that are active, i.e., IgM, IgG, and their isotypes that may be involved in switching isotype. Besides, the irrelevant Simpson index (D) recommends a diverse immune reaction that is conceivable as a subunit vaccine contains various $\mathrm{B}$-cell and $\mathrm{T}$-cell epitopes.

The translation efficiency of foreign genes inside the host system varies because of the incompatibility of mRNA codons, which require codon optimization for higher expression [92]. CAI value obtained was 1.0 and GC content (53.2\%) was also within the optimum limit suggesting possible higher expression in the E. coli $\mathrm{K}-12$ system. The main aim of MESV in silico cloning was to direct genetic engineers and molecular biologists on the expected expression level and the potential cloning sites in a particular expression system i.e., E. coli $\mathrm{K} 12$ system.

We applied the next-generation vaccine designing approach in this research to create a MESV construct, capable of generating immunological responses against the SARS-CoV-2. We believe that our vaccine will successfully produce humoral and cell-mediated immune responses. Interaction and binding patterns between receptor and vaccine protein were stable and higher. Moreover, in immune simulation, effective immune responses were observed in real life. Thus, MESV designed carefully using such a methodology could become an important asset in combating viral infections.

Computational/immunoinformatics approaches rely on experimental methodologies to generate initial raw data for further analyses. The data quality and efficiency of computational algorithms being applied, can limit the accuracy of immunoinformatics predictions. Therefore, further in vivo and in vitro investigations are however required to ensure the real potential of designed MESV to combat COVID-19.

\section{Conclusions}

Taken together, we characterized SARS-CoV-2 structural proteins $(\mathrm{S}, \mathrm{E}$, and $\mathrm{M})$ for antigenic epitopes and proposed a potential MESV utilizing various immunoinformatics and computational approaches. The findings of this research could save time and related costs for the study of experimental epitope targets. The MESV can activate all host immune system components and has adequate physicochemical and structural properties. It also appears to interact very stably with an innate immune receptor TLR3, making it more likely to be introduced into the host immune system. To reveal its effectiveness in the fight against COVID-19, however, additional in vitro and in vivo experiments are warranted. 


\section{Supplementary information}

Supplementary information accompanies this paper at https://doi.org/10. 1186/s40249-020-00752-w

Additional file 1: Figure S1. 3D structural representation of SARS-CoV2 structural proteins: (A) S protein, (B) E protein and (C) M protein. Figure S2. (a) the E protein contains a-helix $(77.33 \%, 58)$ and random coil $(22.66 \%, 17)$; (b) the $z$-score $(0.41)$ of the E protein; (c) the Ramachandran plot of refined structure shows 97.3, 2.7 and $0.0 \%$ residues in favored, allowed and disallowed region, respectively. Figure S3. (a) the M protein contains a-helix $(40.54 \%, 90), \beta$-strand $(24.32 \%, 54)$ and random coil $(35.13 \%, 78)$; (b) the $z$-score $(-3.88)$ of the M protein; (c) the Ramachandran plot of refined structure shows $96.8,2.7$ and $0.5 \%$ residues in favored, allowed and disallowed region, respectively. Figure S4. Specific sites of B cells predicted linear epitopes on the 3D structure of SARS-CoV2 proteins: (A) S protein, (B) E protein and (C) M protein. Figure S5. (A) Prediction of antigenic determinants of $S$ proteinusing Kolaskar and Tongaonkar antigenicity scale; (B) Beta Turns analyses in S protein using Chou and Fasman Beta Turn prediction; (C) Hydrophilicity Prediction of S protein using Parker Hydrophilicity; (D) Surface Accessibility Analyses of S protein using Emini Surface Accessibility Scale; (E) Flexibility Analyses of S protein using Karplus and Schulz Flexibility Scale. Figure S6. (A) Prediction of antigenic determinants of E protein using Kolaskar and Tongaonkar antigenicity scale; (B) Beta Turns Analyses in E protein using Chou and Fasman Beta Turn Prediction; (C) Hydrophilicity Prediction of E protein using Parker Hydrophilicity; (D) surface accessibility analyses of E protein using Emini Surface Accessibility Scale; (E) Flexibility Analyses of E protein using Karplus and Schulz Flexibility Scale. Figure S7. (A) Prediction of antigenic determinants of $\mathrm{M}$ protein using Kolaskar and Tongaonkar Antigenicity Scale; (B) Beta turns analyses in M protein using Chou and Fasman Beta Turn Prediction; (C) Hydrophilicity Prediction of M protein using Parker Hydrophilicity; (D) Surface Accessibility Analyses of M protein using Emini Surface Accessibility Scale; (E) Flexibility Analyses of M protein using Karplus and Schulz Flexibility Scale.

Additional file 2: Table S1. Structural details of the SARS-CoV-2 structural protein predicted models.

Additional file 3: Table S2. Linear B cell epitopes predicted through ABCPred 2.0 server (NT: nontoxic).

Additional file 4: Table S3. Emini surface accessibility of SARS-CoV-2 structural proteins.

Additional file 5: Table S4. Discontinuous epitopes predicted through DiscoTop 2.0 server.

Additional file 6: Table S5. MHC class-I allele and MHC class-II binding peptides with their antigenicity scores.

Additional file 7: Table S6. Digestion, allergenicity, toxicity and physiochemical profiling of selected peptides (NA: not allergic; NT: nontoxic).

Additional file 8: Table S7. Linear B cell epitopes predicted in vaccine construct.

Additional file 9: Table S8. Conformational epitopes in 3D structure of vaccine.

\section{Abbreviations}

3D: Three dimensional; CAl: Codon adaptation index; CoV: Coronavirus; COVID-19: Coronavirus disease 2019; CTL: Cytotoxic T lymphocytes; E: Envelope; HLA: Human leukocyte antigen; HTL: Helper T lymphocyte; M: Membrane; MERS-CoV: Middle East respiratory syndrome corona virus; MESV: Multiepitope-based subunit vaccine; MHC: Major histocompatibility complex; PDB: Protein Data Bank; pl: Isoelectric point; S: Spike; SARS-CoV2: Severe acute respiratory syndrome coronavirus 2; TLR3: Toll-like receptors-3

\section{Acknowledgements}

Authors would like to acknowledge Guangxi University and Government College University Faisalabad for providing facilities for this study.

\section{Authors' contributions}

LLC and UAA conceived and designed this study; MTQ and FS performed the experiments; SA, SA, IF, MMF and AZ analyse the results; MTQ and FS wrote the manuscript; LLC and UAA improved and revised the manuscript, and all the authors approved the final version.

\section{Funding}

This work was supported by the Starting Research Grant for High-level Talents from Guangxi University and Postdoctoral research platform grant of Guangxi University.

\section{Availability of data and materials \\ Not applicable.}

Ethics approval and consent to participate

Not applicable.

\section{Consent for publication}

Not applicable.

\section{Competing interests}

All authors have no competing interests.

\section{Author details}

${ }^{1}$ College of Life Science and Technology, Guangxi University, Nanning, P. R. China. ${ }^{2}$ Department of Bioinformatics and Biotechnology, Government College University Faisalabad (GCUF), Faisalabad, Pakistan. ${ }^{3}$ Jinnah Hospital, Lahore, Pakistan. ${ }^{4}$ Department of Healthcare Biotechnology, Atta-ur-Rahman School of Applied Biosciences (ASAB), National University of Sciences and Technology (NUST), Islamabad, Pakistan.

Received: 29 April 2020 Accepted: 8 September 2020

Published online: 16 September 2020

\section{References}

1. Tahir ul Qamar M, Alqahtani SM, Alamri MA, Chen LL. Structural basis of SARS-CoV-2 3CL ${ }^{\text {pro }}$ and anti-COVID-19 drug discovery from medicinal plants. J Pharm Anal. 2020;10(4):313-19.

2. Alamri MA, Tahir ul Qamar M, Mirza MU, Bhadane R, Alqahtani SM, Muneer I, et al. Pharmacoinformatics and molecular dynamics simulation studies reveal potential covalent and FDA-approved inhibitors of SARS-CoV-2 main protease 3CLpro. J Biomol Struct Dyn. 2020:1-13. https://doi.org/10.1080/ 07391102.2020.1782768.

3. Tahir ul Qamar M, Saleem S, Ashfaq UA, Bari A, Anwar F, Alqahtani S. Epitope-based peptide vaccine design and target site depiction against Middle East respiratory syndrome coronavirus: an immune-informatics study. J Transl Med. 2019;17(1):362.

4. de Wilde AH, Snijder EJ, Kikkert M, van Hemert MJ. Host factors in coronavirus replication. Curr Top Microbiol Immunol. 2018;419:1-42.

5. Wang C, Horby PW, Hayden FG, Gao GF. A novel coronavirus outbreak of global health concern. Lancet. 2020;395(10223):470-3.

6. Gralinski LE, Menachery VD. Return of the coronavirus: 2019-nCoV. Viruses. 2020;12(2):135.

7. Peiris JS, Yuen KY, Osterhaus AD, Stöhr K. The severe acute respiratory syndrome. N Engl J Med. 2003;349(25):2431-41.

8. Cotten M, Watson SJ, Zumla Al, Makhdoom HQ, Palser AL, Ong SH, et al. Spread, circulation, and evolution of the Middle East respiratory syndrome coronavirus. MBio. 2014;5(1):e01062-13.

9. Huang C, Wang Y, Li X, Ren L, Zhao J, Hu Y, et al. Clinical features of patients infected with 2019 novel coronavirus in Wuhan, China. Lancet. 2020;395(10223):497-506.

10. Chen Y, Liu Q, Guo D. Coronaviruses: genome structure, replication, and pathogenesis. J Med Virol. 2020;92(4):418-23.

11. Ji W, Wang W, Zhao X, Zai J, Li X. Cross-species transmission of the newly identified coronavirus 2019-nCoV. J Med Virol. 2020;94(4):433-40.

12. Tang $X$, Wu C, Li X, Song $Y$, Yao $X, W u X$, et al. On the origin and continuing evolution of SARS-CoV-2. Natl Sci Rev. 2020;6:6.

13. Zhang $Y Z$, Holmes EC. A genomic perspective on the origin and emergence of SARS-CoV-2. Cell. 2020;181(2):223-7.

14. Shammah A, Budoor H, Suad M, Richard K, Maha S, Asokan G. Middle East respiratory syndrome corona virus (MERS-CoV): Levels of knowledge and 
awareness in Bahrain. KnE Life Sciences. 2018;4:98-114. https://doi.org/10. 18502/kls.v4i6.3094.

15. AP K, VS A. Design of multi-epitope vaccine candidate against SARS-CoV-2: a in-silico study. J Biomol Struct Dyn. 2020:1-9. https://doi.org/10.1080/ 07391102.2020 .1770127$.

16. Zhou Y, Hou Y, Shen J, Huang Y, Martin W, Cheng F. Network-based drug repurposing for novel coronavirus 2019-nCoV/SARS-CoV-2. Cell Discov. 2020,6(1):1-18.

17. Lythgoe MP, Middleton P. Ongoing clinical trials for the management of the COVID-19 pandemic. Trends Pharmacol Sci. 2020;41(6):363-82.

18. Biopharmaceuticals C. Clover Initiates Development of Recombinant Subunit-Trimer Vaccine for Coronavirus (2019-nCoV). http://www. cloverbiopharma.com/index.php?m=content\&c=index\&a=show\&catid=11 \&id $=40$. Accessed 2 Mar 2020.

19. Liu J, Cao R, Xu M, Wang X, Zhang H, Hu H, et al. Hydroxychloroquine, a less toxic derivative of chloroquine, is effective in inhibiting SARS-CoV-2 infection in vitro. Cell Discov. 2020;6(1):1-4.

20. Wang M, Cao R, Zhang L, Yang X, Liu J, Xu M, et al. Remdesivir and chloroquine effectively inhibit the recently emerged novel coronavirus (2019-nCoV) in vitro. Cell Res. 2020;30(3):269-71.

21. Horby P, Lim WS, Emberson J, Mafham M, Bell J, Linsell L, et al. Effect of Dexamethasone in Hospitalized Patients with COVID-19: Preliminary Report. medRxiv. 2020. https://doi.org/10.1101/2020.06.22.20137273.

22. Mukherjee $\mathrm{S}$. The first coronavirus drug candidate is set for testing in China. Fortune. 2020. https://fortune.com/2020/02/03/coronavirus-vaccine-testingin-china/. Accessed 2 Mar 2020.

23. Chen W-H, Strych U, Hotez PJ, Bottazzi ME. The SARS-CoV-2 vaccine pipeline: an overview. Curr Trop Med Rep. 2020:1-4. https://doi.org/10.1007/ s40475-020-00201-6.

24. Roper RL, Rehm KE. SARS vaccines: where are we? Expert Rev Vaccines. 2009;8(7):887-98.

25. Prompetchara E, Ketloy C, Palaga T. Immune responses in COVID-19 and potential vaccines: lessons learned from SARS and MERS epidemic. Asian Pac J Allergy Immunol. 2020;38(1):1-9.

26. Le TT, Andreadakis Z, Kumar A, Roman RG, Tollefsen S, Saville M, et al. The COVD-19 vaccine development landscape. Nat Rev Drug Discov. 2020;19(5):305-6.

27. Tahir ul Qamar M, Rehman A, Ashfaq UA, Awan MQ, Fatima I, Shahid F, et al. Designing of a next generation multiepitope based vaccine (MEV) against SARS-COV-2: Immunoinformatics and in silico approaches. BioRxiv. 2020. https://doi.org/10.1101/2020.02.28.970343.

28. Baruah V, Bose S. Immunoinformatics-aided identification of $\mathrm{T}$ cell and B cell epitopes in the surface glycoprotein of 2019-nCoV. J Med Virol. 2020;92(5): 495-500.

29. Tahir ul Qamar M, Shokat Z, Muneer I, Ashfaq UA, Javed H, Anwar F, et al. Multiepitope-based subunit vaccine design and evaluation against respiratory syncytial virus using reverse vaccinology approach. Vaccines. 2020;8(2):288.

30. Tahir ul Qamar M, Bari A, Adeel MM, Maryam A, Ashfaq UA, Du X, et al. Peptide vaccine against chikungunya virus: immuno-informatics combined with molecular docking approach. J Transl Med. 2018;16(1):298.

31. Ahmad B, Ashfaq UA, Rahman MU, Masoud MS, Yousaf MZ. Conserved B and T cell epitopes prediction of ebola virus glycoprotein for vaccine development: an immuno-informatics approach. Microb Pathog. 2019;132:243-53.

32. Cockrell AS, Johnson JC, Moore IN, Liu DX, Bock KW, Douglas MG, et al. A spike-modified Middle East respiratory syndrome coronavirus (MERS-CoV) infectious clone elicits mild respiratory disease in infected rhesus macaques. Sci Rep. 2018:8(1):10727.

33. Amer H, Alqahtani AS, Alaklobi F, Altayeb J, Memish ZA. Healthcare worker exposure to Middle East respiratory syndrome coronavirus (MERS-CoV): revision of screening strategies urgently needed. Int J Infect Dis. 2018;71:113-6.

34. Hui DS, Azhar El, Kim YJ, Memish ZA, Oh MD, Zumla A. Middle East respiratory syndrome coronavirus: risk factors and determinants of primary, household, and nosocomial transmission. Lancet Infect Dis. 2018;18(8):e217-27.

35. Ashfaq UA, Ahmed B. De novo structural modeling and conserved epitopes prediction of Zika virus envelop protein for vaccine development. Viral Immunol. 2016;29(7):436-43.

36. Benson DA, Karsch-Mizrachi I, Lipman DJ, Ostell J, Sayers EW. GenBank Nucleic Acids Res. 2008;37(suppl1):D26-31.

37. Doytchinova IA, Flower DR. VaxiJen: a server for prediction of protective antigens, tumour antigens and subunit vaccines. BMC Bioinformatics. 2007;8(1):4.

38. Dimitrov I, Flower DR, Doytchinova I. AllerTOP-a server for in silico prediction of allergens. BMC Bioinformatics. 2013;14(Suppl 6):S4.
39. Berman $\mathrm{H}$, Henrick K, Nakamura $\mathrm{H}$. Announcing the worldwide protein data bank. Nat Struct Mol Biol. 2003;10(12):980

40. Webb B, Sali A. Protein structure modeling with MODELLER. Methods Mol Biol. 2014;1137:1-15.

41. Pettersen EF, Goddard TD, Huang CC, Couch GS, Greenblatt DM, Meng EC, et al. UCSF chimera - a visualization system for exploratory research and analysis. J Comput Chem. 2004;25(13):1605-12.

42. Shin W-H, Lee GR, Heo L, Lee H, Seok C. Prediction of protein structure and interaction by GALAXY protein modeling programs. Bio Design. 2014;2(1):1-11.

43. Xu D, Zhang Y. Improving the physical realism and structural accuracy of protein models by a two-step atomic-level energy minimization. Biophys J. 2011;101(10):2525-34.

44. Wiederstein M, Sippl MJ. ProSA-web: interactive web service for the recognition of errors in three-dimensional structures of proteins. Nucleic Acids Res. 2007;35(suppl_2):W407-10.

45. Lovell SC, Davis IW, Arendall WB III, De Bakker PI, Word JM, Prisant MG, et al. Structure validation by $C a$ geometry: $\varphi, \psi$ and $C \beta$ deviation. Proteins. 2003; 50(3):437-50.

46. Saha S, Raghava GPS. Prediction of continuous B-cell epitopes in an antigen using recurrent neural network. Proteins. 2006;65(1):40-8.

47. Fieser TM, Tainer JA, Geysen HM, Houghten RA, Lerner RA. Influence of protein flexibility and peptide conformation on reactivity of monoclonal anti-peptide antibodies with a protein alpha-helix. Proc Natl Acad Sci U S A. 1987;84(23):8568-72

48. Jespersen MC, Peters B, Nielsen M, Marcatili P. BepiPred-2.0: improving sequence-based B-cell epitope prediction using conformational epitopes. Nucleic Acids Res. 2017:45(W1):W24-9.

49. Sun P, Ju H, Liu Z, Ning Q, Zhang J, Zhao X, et al. Bioinformatics resources and tools for conformational B-cell epitope prediction. Comput Math Methods Med. 2013;2013:943636.

50. DeLano WL. Pymol: An open-source molecular graphics tool. CCP4 Newsl Protein Protein Crystallogr. 2002;40(1):82-92.

51. Zhang M, Ishii K, Hisaeda H, Murata S, Chiba T, Tanaka K, et al. Ubiquitinfusion degradation pathway plays an indispensable role in naked DNA vaccination with a chimeric gene encoding a syngeneic cytotoxic T lymphocyte epitope of melanocyte and green fluorescent protein. Immunology. 2004;112(4):567-74.

52. Dimitrov I, Naneva L, Doytchinova I, Bangov I. AllergenFP: allergenicity prediction by descriptor fingerprints. Bioinformatics. 2014;30(6):846-51.

53. Gupta S, Kapoor P, Chaudhary K, Gautam A, Kumar R, Raghava GP, et al. In silico approach for predicting toxicity of peptides and proteins. PLoS One. 2013;8(9):e73957.

54. Bui H-H, Sidney J, Li W, Fusseder N, Sette A. Development of an epitope conservancy analysis tool to facilitate the design of epitope-based diagnostics and vaccines. BMC Bioinformatics. 2007;8(1):361.

55. Adhikari UK, Tayebi M, Rahman MM. Immunoinformatics approach for epitope-based peptide vaccine design and active site prediction against polyprotein of emerging Oropouche virus. J Immunol Res. 2018;2018: 6718083.

56. Bui H-H, Sidney J, Dinh K, Southwood S, Newman MJ, Sette A. Predicting population coverage of T-cell epitope-based diagnostics and vaccines. BMC Bioinformatics. 2006;7(1):153.

57. Hoover DM, Wu Z, Tucker K, Lu W, Lubkowski J. Antimicrobial characterization of human $\beta$-defensin 3 derivatives. Antimicrob Agents Chemother. 2003:47(9):2804-9.

58. Mahram A, Herbordt MC. Fast and accurate NCBI BLASTP: acceleration with multiphase FPGA-based prefiltering, Proceedings of the 24th ACM International Conference on Supercomputing; 2010. p. 73-82.

59. Gasteiger E, Hoogland C, Gattiker A, Duvaud S, Wilkins MR, Appel RD, Bairoch A. Protein identification and analysis tools on the ExPASy server. In: Walker JM, editor. The Proteomics Protocols Handbook. Totowa: Humana Press; 2005.

60. Bjellqvist B, Hughes GJ, Pasquali C, Paquet N, Ravier F, Sanchez JC, et al. The focusing positions of polypeptides in immobilized $\mathrm{pH}$ gradients can be predicted from their amino acid sequences. J Electrophoresis. 1993;14(1): 1023-31.

61. Blaszczyk M, Jamroz M, Kmiecik S, Kolinski A. CABS-fold: server for the de novo and consensus-based prediction of protein structure. Nucleic Acids Res. 2013;41(W1):W406-11.

62. Heo L, Park H, Seok C. GalaxyRefine: Protein structure refinement driven by side-chain repacking. Nucleic Acids Res. 2013;41(Web Server issue):W384-8. 
63. Lengths M, Angles M. Limitations of structure evaluation tools errat. Quick Guideline Comput Drug Des. 2018;16:75

64. Ponomarenko J, Bui H-H, Li W, Fusseder N, Bourne PE, Sette A, et al. ElliPro: a new structure-based tool for the prediction of antibody epitopes. BMC Bioinformatics. 2008;9(1):514.

65. Tovchigrechko A, Vakser IA. GRAMM-X public web server for proteinprotein docking. Nucleic Acids Res. 2006;34(suppl_2):W310-4.

66. Laskowski RA, Jabłońska J, Pravda L, Vařeková RS, Thornton JM. PDBsum: structural summaries of PDB entries. Protein Sci. 2018;27(1):129-34.

67. Rapin N, Lund O, Bernaschi M, Castiglione F. Computational immunology meets bioinformatics: the use of prediction tools for molecular binding in the simulation of the immune system. PLoS One. 2010;5(4):e9862.

68. Smith CL, Econome JG, Schutt A, Klco S, Cantor CR. A physical map of the Escherichia coli K12 genome. Science. 1987;236(4807):1448-53.

69. Grote A, Hiller K, Scheer M, Munch R, Nortemann B, Hempel DC, et al. JCat: a novel tool to adapt codon usage of a target gene to its potential expression host. Nucleic Acids Res. 2005;33(Web Server issue):W526-31.

70. Sharp PM, Li WH. The codon adaptation index--a measure of directional synonymous codon usage bias, and its potential applications. Nucleic Acids Res. 1987;15(3):1281-95.

71. Källberg M, Wang H, Wang S, Peng J, Wang Z, Lu H, et al. Template-based protein structure modeling using the RaptorX web server. Nat Protoc. 2012; 7(8):1511-22.

72. Pandey RK, Narula A, Naskar M, Srivastava S, Verma P, Malik R, et al. Exploring dual inhibitory role of febrifugine analogues against Plasmodium utilizing structure-based virtual screening and molecular dynamic simulation. J Biomol Struct Dyn. 2017;35(4):791-804.

73. Douglas MG, Kocher JF, Scobey T, Baric RS, Cockrell AS. Adaptive evolution influences the infectious dose of MERS-CoV necessary to achieve severe respiratory disease. Virology. 2018;517:98-107.

74. Nain Z, Karim MM, Sen MK, Adhikari UK. Structural basis and designing of peptide vaccine using PE-PGRS family protein of Mycobacterium ulcerans-an integrated vaccinomics approach. Mol Immunol. 2020;120:146-63.

75. Song HC, Seo MY, Stadler K, Yoo BJ, Choo QL, Coates SR, et al. Synthesis and characterization of a native, oligomeric form of recombinant severe acute respiratory syndrome coronavirus spike glycoprotein. J Virol. 2004; 78(19):10328-35.

76. Kirchdoerfer RN, Cottrell CA, Wang N, Pallesen J, Yassine HM, Turner HL, et al. Pre-fusion structure of a human coronavirus spike protein. Nature. 2016:531(7592):118-21

77. Siu Y, Teoh K, Lo J, Chan C, Kien F, Escriou N, et al. The M, E, and N structural proteins of the severe acute respiratory syndrome coronavirus are required for efficient assembly, trafficking, and release of virus-like particles. J Virol. 2008;82(22):11318-30.

78. J Alsaadi EA, Jones IM. Membrane binding proteins of coronaviruses. Future Virol. 2019;14(4):275-86.

79. Schoeman D, Fielding BC. Coronavirus envelope protein: current knowledge. Virol J. 2019;16(1):69.

80. Zhang L. Multi-epitope vaccines: a promising strategy against tumors and viral infections. Cell Mol Immunol. 2018;15(2):182-4.

81. Abdelmageed Ml, Abdelmoneim AH, Mustafa MI, Elfadol NM, Murshed NS, Shantier SW, et al. Design of a Multiepitope-Based Peptide Vaccine against the E protein of human COVID-19: an Immunoinformatics approach. Biomed Res Int. 2020;2020:2683286.

82. Bhattacharya M, Sharma AR, Patra P, Ghosh P, Sharma G, Patra BC, et al. Development of epitope-based peptide vaccine against novel coronavirus 2019 (SARS-COV-2): Immunoinformatics approach. J Med Virol. 2020;92(6):618-31.

83. Mishra S. T cell epitope-based vaccine design for pandemic novel coronavirus 2019-nCoV. chemrxiv; 2020

84. Cooper NR, Nemerow GR. The role of antibody and complement in the control of viral infections. J Invest Dermatol. 1984:83(s 1):121-7.

85. Bacchetta R, Gregori S, Roncarolo MG. CD4+ regulatory T cells: mechanisms of induction and effector function. Autoimmun Rev. 2005:4(8):491-6.

86. Pohl R, Gilman R, Miller GA, Pachucki K. Muonic hydrogen and the proton radius puzzle. Annu Rev Nucl Part Sci. 2013;63:175-204.

87. Meza B, Ascencio F, Sierra-Beltrán AP, Torres J, Angulo C. A novel design of a multi-antigenic, multistage and multi-epitope vaccine against helicobacter pylori: an in silico approach. Infect Genet Evol. 2017;49:309-17.

88. Lee S, Nguyen MT. Recent advances of vaccine adjuvants for infectious diseases. Immune Netw. 2015;15(2):51-7.
89. Arai R, Ueda H, Kitayama A, Kamiya N, Nagamune T. Design of the linkers which effectively separate domains of a bifunctional fusion protein. Protein Eng. 2001;14(8):529-32.

90. Tahir ul Qamar M, Maryam A, Muneer I, Xing F, Ashfaq UA, Khan FA, et al. Computational screening of medicinal plant phytochemicals to discover potent pan-serotype inhibitors against denque virus. Sci Rep. 2019;9(1):1-16.

91. Durdagi S, Tahir ul Qamar MT, Salmas RE, Tariq Q, Anwar F, Ashfaq UA. Investigating the molecular mechanism of staphylococcal DNA gyrase inhibitors: a combined ligand-based and structure-based resources pipeline. J Mol Graph Model. 2018;85:122-9.

92. Pandey RK, Bhatt TK, Prajapati VK. Novel immunoinformatics approaches to design multi-epitope subunit vaccine for malaria by investigating anopheles salivary protein. Sci Rep. 2018;8(1):1-11.
Ready to submit your research? Choose BMC and benefit from:

- fast, convenient online submission

- thorough peer review by experienced researchers in your field

- rapid publication on acceptance

- support for research data, including large and complex data types

- gold Open Access which fosters wider collaboration and increased citations

- maximum visibility for your research: over $100 \mathrm{M}$ website views per year

At BMC, research is always in progress.

Learn more biomedcentral.com/submissions 